\title{
Vaginal support pessary in treatment of rectal prolapse Case series and review
}

\author{
Myriam Girgis ${ }^{1}$, Joseph Lee ${ }^{1}$, and Alan Meagher ${ }^{1}$ \\ ${ }^{1}$ St Vincent's Hospital
}

June 9, 2020

\begin{abstract}
Rectal prolapse is a pelvic floor disorder that involves descent of full or partial thickness rectum through anus. The novel use of a vaginal support pessary may avoid surgery in those who are not fit for surgery or prefer to avoid surgery, and may provide temporary relief whilst awaiting surgery.
\end{abstract}

\section{Cases}

A 98 year-old female from a nursing home, presented with recurrent symptomatic rectal prolapse after a Delorme's procedure seven months prior. Her past medical history includes Alzheimer's disease and vascular dementia, hypertension, hypercholesterolaemia, congenital cardiac failure, paroxysmal atrial fibrillation, osteoporosis and recurrent falls.

Despite being considered for abdominal rectopexy, urogynaecology review was sought for second opinion. There was no vaginal prolapse on examination. An oval vaginal pessary was trialled (Figure 1). She is currently on vaginal estrogen cream twice per week with a plan to change the pessary every 2-3 months. Eight months later, the patient continues to comfortably open her bowels without rectal prolapse or vaginal discharge or bleeding.

The second patient is a 78 year-old woman who presented with severe rectal pain, recurrent rectal prolapse, constipation and associated urinary retention.

Past medical history included a Delorme's procedure 3 years ago, anterior vaginal wall repair, refractory chronic idiopathic thrombocytopenia purpura secondary to splenectomy, degenerative spinal disease with canal stenosis and neuropathy requiring laminectomy and spinal fusion, dyslipidaemia, asthma, Grave's disease, osteoporosis, osteoarthritis and recurrent falls.

On examination, the patient had a $4 \mathrm{~cm}$ rectal prolapse outside the anal verge, without a vaginal prolapse. A size 4 Sayco oval pessary with support was fitted. Four months after placement, the patient reported good bowel motions, nil rectal prolapse recurrence, nil urinary retention and nil vaginal discharge or bleeding.

The third patient is a 93 year-old woman who presented with recurrence of her rectal prolapse following a Delorme's procedure two years ago. Her rectal prolapse did not recur since pessary insertion 6 months ago.

\section{Discussion}

Rectal prolapse has an incidence of $0.5 \%$ and is more commonly seen in the elderly ${ }^{3}$. Risk factors include female gender, age over 40, multiparity, vaginal delivery, prior pelvic surgery, chronic straining, chronic diarrhoea or constipation, cystic fibrosis, dementia or stroke, pelvic floor dysfunction and anatomical defects such as anterior or posterior vaginal wall prolapse ${ }^{1}$. 
Patients present with abdominal discomfort, incomplete bowel evacuation and mucus and/or stool discharge, altered bowel habits, faecal incontinence and a prolapsing mass through the anus that may reduce spontaneously or require manual reduction ${ }^{1,3}$.

It is associated with a number of anatomical anomalies including diastasis of the levator ani, a deep cul-de-sac, a redundant sigmoid colon, a patulous anal sphincter, and a loss of rectal sacral attachments ${ }^{3}$.

Initial assessment should include a complete history and physical examination ${ }^{3}$. The clinician should review the patient's diet, fiber intake, fluid intake, and use of medication that may contribute to constipation, symptoms of concomitant uterine or vaginal prolapse and urinary incontinence ${ }^{1}$, and underlying conditions ${ }^{3}$.

On examination, the concentric rings of the rectum protruding through the anus are the hallmark of rectal prolapse $^{1}$. Digital rectal examination may find a patulous anus, poor sphincter tone, mass and concomitant pelvic floor pathology ${ }^{1}$.

Defecography with fluoroscopy or MRI is helpful if the prolapse is unable to be reproduced on examination and may reveal concomitant pelvic floor prolapse ${ }^{1}$. Those with pelvic floor prolapse may benefit from urodynamics $^{3}$. A colonoscopy should be considered to look for an underlying neoplasm ${ }^{3}$. Anal physiologic testing may identify associated functional disorders such as constipation or fecal incontinence ${ }^{3}$.

The general consensus in the literature is that rectal prolapse cannot be corrected non-surgically with medical therapy alone ${ }^{3}$. Medical therapy to date is limited to adequate fluid and fibres, stool softeners to treat constipation and skin care to avoid maceration ${ }^{1,3}$; the use of anal plugs have been described ${ }^{4}$. These are considered palliative interventions that do not address the prolapse itself but may improve the patient's quality of life ${ }^{3}$.

Surgical repair of rectal prolapse is the treatment of choice $^{1}$, either transabdominal or perineal. There is no consensus on the optimal surgical approach in terms of recurrence rate or bowel function ${ }^{1}$. Choice of surgery depends on the patient's status, age, baseline bowel function, surgeon's experience and preference ${ }^{1}$.

Abdominal procedures have higher mortality and morbidity rates compared to perineal procedures ${ }^{2}$. The mortality rates for abdominal procedures is 0 to 7 percent and morbidity rates is 0 to 52 percent $^{2}$. The most common major complications include pelvic infection, hematomas, fistulas, stenosis and obstructed defecation ${ }^{2}$. As such, patients with poorer medico-surgical co-morbidities may be more suitable for perineal repairs ${ }^{2}$.

Although abdominal procedures have lower recurrence rates, perineal procedures have fewer risks and complications than abdominal procedures with reported mortality 0 to 4 percent and morbidity rates less than 20 percent $^{2}$. Complications after a perineal repair include bleeding, pelvic infection and faecal leakage ${ }^{2}$.

After surgery, symptoms of faecal incontinence generally improve; symptoms of constipation generally improve with perineal procedures but may improve or worsen with abdominal procedures ${ }^{2}$. Patients undergoing perineal repair procedures show an improvement in constipation ranging from 13 to 100 percent $^{2}$.

Vaginal pessaries are commonly used in gynaecological practice as a non-surgical option for the treatment of vaginal pelvic organ prolapse (POP) and stress urinary incontinence ${ }^{5}$.

There are reports of intravaginal devices for faecal incontinence treatment ${ }^{8}$. This 'vaginal bowel-control system' is described as a vaginal silicone-coated stainless steel base with a posterior balloon that is pressureregulated pump ${ }^{8}$. The pump is deflated for a bowel movement and inflated to prevent incontinence; this acts on the distal rectum to allow the patient to control her bowel motions ${ }^{8}$. Similar to the vaginal pessary for prolapse, not all women could be successfully fit as a result of discomfort, vaginal dimensions or exclusion criteria $^{8}$.

Vaginal pessaries are generally made of medical grade silicone that come in a range of shapes and sizes to support the pelvic organs, with the space occupying type being more suitable for a larger prolapse ${ }^{5}$. There is no robust evidence for the use of one type of pessary over another, so similar to the pessary fitting for POP, 
choice of vaginal pessary for rectal prolapse requires some trial and error ${ }^{5}$. When choosing an initial pessary size, a pelvic examination is required to assess the length of the vagina from the posterior fornix to the pubic symphysis, and to assess the vaginal width by spreading the index and middle fingers horizontally at the level of cervix or vaginal vault ${ }^{5}$. Support pessaries lie along the vaginal axis; the posterior component sits in the posterior vaginal fornix and the anterior component sits under the symphysis pubis ${ }^{5,6}$. An appropriate size is achieved when the patient's symptoms are relieved, they are able to void and defecate, the pessary remains in situ with activities of daily living, is comfortable for the patient and allows the patient the preservation of sexual intercourse should this be their wish ${ }^{5}$.

Figure 2 shows a rectocele and intussusception before and after a vaginal pessary is placed in situ, with a reduction of the rectal prolapse possibly by elevating and supporting the previous Delormes "doughnut".

When counselling of patients on use of the vaginal pessary for rectal prolapse, potential complications need to be discussed ${ }^{5}$. Side effects include vaginal discharge, ulcerations, bleeding, pain, vaginal and urinary tract infections, urinary retention, faecal impaction, interference with sexual intercourse, de novo (occult) SUI and pessary expulsion ${ }^{5,6}$. A forgotten pessary can result in erosion into the bowel, bladder or vaginal wall and fistula formation ${ }^{5}$.

Once the pessary is inserted, the woman is asked to strain and cough, ambulate and void and strain while sitting on a toilet ${ }^{6}$. This determines that the pessary will not be expelled and that the patient can void when she returns home ${ }^{5,6}$. Vaginal estrogen should be prescribed for postmenopausal women to reduce the risk of vaginal erosion ${ }^{5}$. There is a need for regular follow-up every 3-6 months to change the pessary, assess for side effects and examine the vagina for erosion ${ }^{5}$.

The success rate, that is the continued use of the pessary in women for POP, is around $80 \%$ at $2-3$ months and $60 \%$ at 6-12 months $s^{5,6}$. 50 to $60 \%$ of women who trial a pessary for POP continue to use them for a further one to two years ${ }^{6,7}$. Long term data on success rate of the use of the pessary for rectal procidentia requires larger trials.

\section{Conclusion:}

We reported three patient anecdotes whereby the vaginal support pessary improved their rectal prolapse and its' associated symptoms, and by inference their quality of life. We believe this to be a first reported series of patients with rectal prolapse responding to vaginal support pessary. Although this novel treatment option could be considered in appropriately selected patients, robust information on its efficacy and patient acceptance needs to be determined in larger series or comparative trials ${ }^{8}$.

\section{Author Contribution:}

Dr. Myriam Girgis: Collected the data, performed literature review and wrote the manuscript.

Prof. Joseph K-S Lee: Collected the data and reviewed and edited the manuscript.

Mr. Alan Meagher: Reviewed and edited the manuscript.

\section{References:}

1. Varma MD, Steele, SR. Overview of rectal procidentia (rectal prolapse). In: Post TW, ed. UpToDate [database on the Internet]. Waltham (MA): UpToDate; 2019 [cited 16 Nov 2019]. Available from: https://www.uptodate.com

2. Varma MD, Steele, SR. Surgical approach to rectal procidentia (rectal prolapse). Post TW, ed. UpToDate [database on the Internet]. Waltham (MA): UpToDate; 2019 [cited 14 Dec 2019]. Available from: https://www.uptodate.com.

3. Bordeianou L, Paquette I, Johnson E, Holubar SD, Gaertner W, Feingold DL, et al. Clinical Practice guidelines for the treatment of rectal prolapse. Dis Colon Rectum [Internet]. 2017 [cited 2020 Jan 01];60(11):1121-1131. Available from: https://www.ncbi.nlm.nih.gov/pubmed/28991074 doi:10.1097/DCR.000000000000889. 
4. Pares D, Vial M, Grande L. An alternative management for high-risk patients with rectal prolapse. Colorectal Dis [Internet]. 2009 Jun [cited 16 Dec 2019];11(5)531-2. Available from: https://www.ncbi.nlm.nih.gov/pubmed/19055516 doi: 10.1111/j.1463-1318.2008.01745.x.

5. Thakar, R. Pessaries for treatment of pelvic organ prolapse.Glob. libr. women's med . [Internet]. 2014 [cited 11 Jan 2020]. Available from: https://www.glowm.com/section_view/heading/Pessaries\%20for\%20Treatment\%20of\%20Pelvic\%20Organ\%20Prolapse/item/805 doi: 10.3843/GLOWM.10479

6. Clemons JF. Vaginal pessaries: Indications, devices, and approach to selection. Post TW, ed. UpToDate [database on the Internet]. Waltham (MA): UpToDate; 2019 [cited 16 Dec 2019]. Available from: https://www.uptodate.com.

7. Rogers GR, Fashokun, TB. Pelvic organ prolapse in women: Epidemiology, risk factors, clinical manifestations, and management. Post TW, ed. UpToDate [database on the Internet]. Waltham (MA): UpToDate; 2019 [cited 10 Jan 2020]. Available from: https://www.uptodate.com.

8. Richter ER, Matthews CA, Muir T, Takase-Sanchez MM, Hale DS, Van Drie D, et al. A Vaginal Bowel-Control System for the Treatment of Faecal Incontinence. Obstet Gynecal [Internet]. 2015 Mar [cited 11 Jan 2020];125(2):540-7. Available from: https://www.ncbi.nlm.nih.gov/pubmed/25730213 doi: 10.1097/AOG.0000000000000639.

Figure 1: Vaginal pessary with support, oval (right), ring (left)

Figure 2a: Sagittal view, Perineal USS: at rest \& Valsalva - rectocele and intussusception

Figure 2b: Sagittal view, Perineal USS: reduction of rectocele, intussusception with vaginal pessary
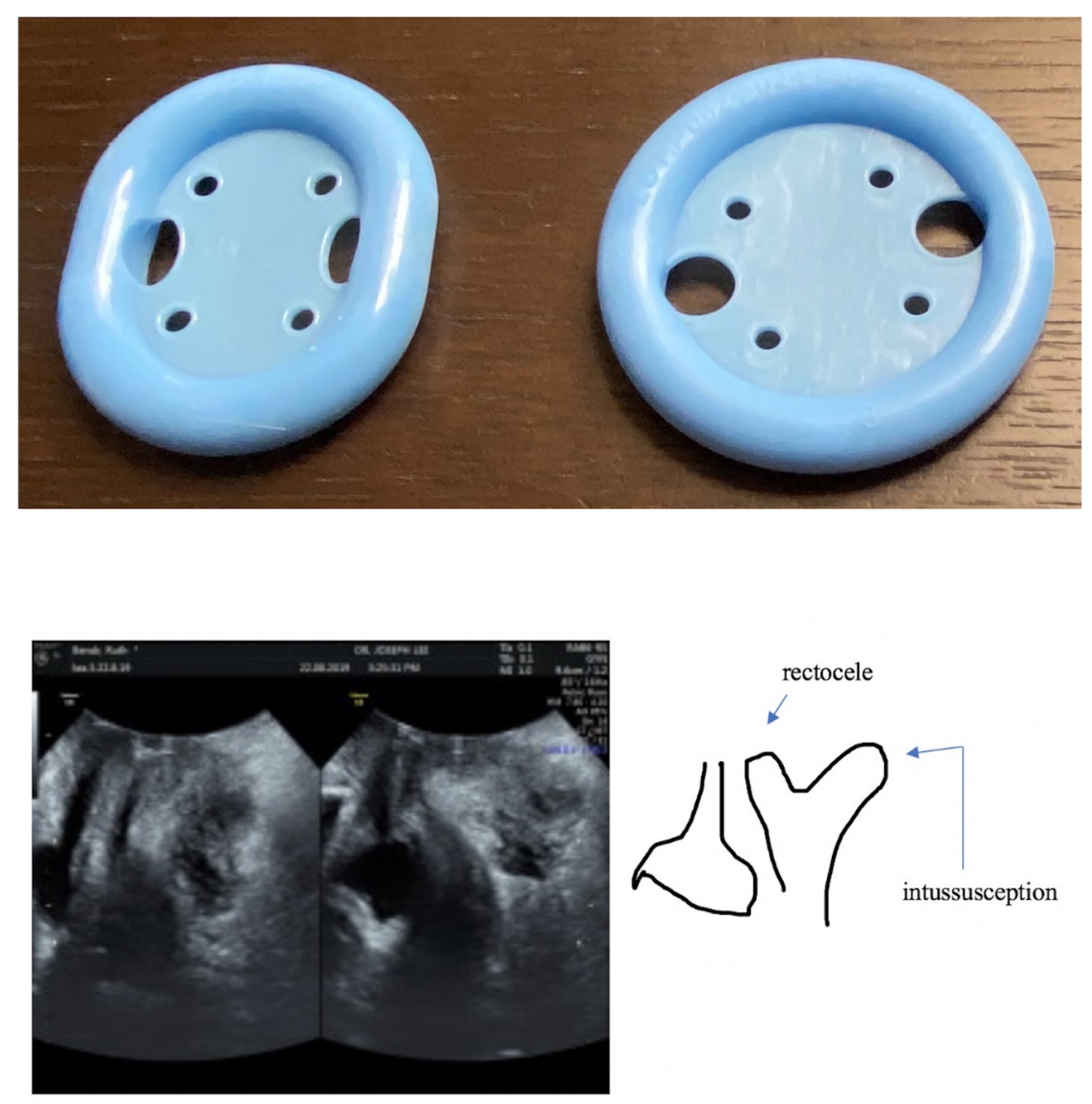

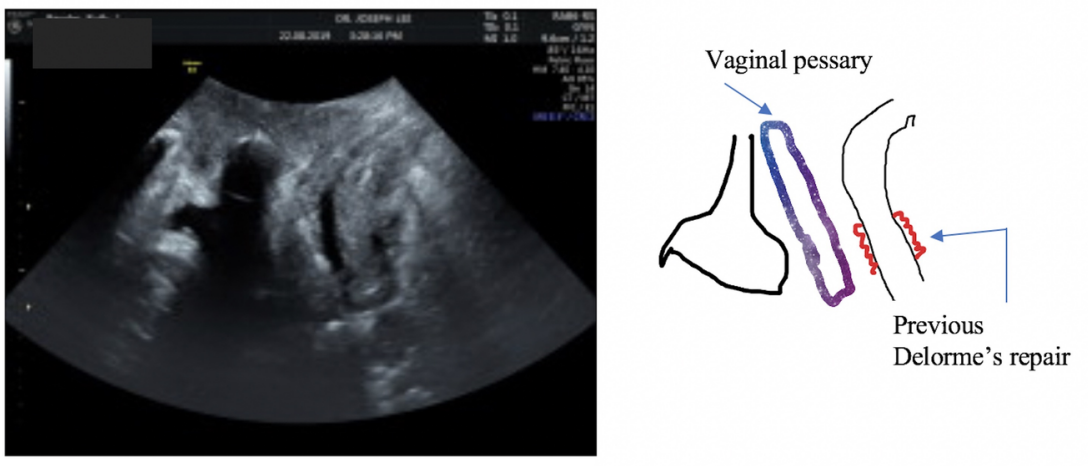